\title{
The mass-conserving domain decomposition method for convection diffusion equations with variable coefficients
}

\author{
Ruiqi Dong ${ }^{1}$, Zhongguo Zhou ${ }^{1}$, Xiangdong Chen ${ }^{1}$, Huiguo Tang ${ }^{1}$, and Qi Zhang ${ }^{1}$ \\ ${ }^{1}$ Shandong Agricultural University
}

March 6, 2021

\begin{abstract}
In this paper, a conserved domain decomposition method for solving convection-diffusion equations with variable coefficients is analyzed. The interface fluxes over the sub-domains are firstly obtained by the explicit fluxes scheme. Secondly, the interior solutions and fluxes over each sub-domains are computed by the modified upwind implicit scheme. Then, the interface fluxes are corrected by the obtained solutions. We prove rigorously that our scheme is mass conservative, unconditionally stable and of second-order convergence in spatial step. Numerical examples test the theoretical analysis and efficiencies. Lastly, we extend our scheme to the nonlinear convection-diffusion equations and give the error estimate.
\end{abstract}

\section{Hosted file}

cam4_new.pdf available at https://authorea.com/users/399874/articles/512283-the-massconserving-domain-decomposition-method-for-convection-diffusion-equations-with-variablecoefficients 